Scalable Computing: Practice and Experience, ISSN 1895-1767, http://www.scpe.org

(C) 2020 SCPE.

Volume 21, Issue 2, pp. 157-158, DOI 10.12694:/scpe.v21i2.1581

\title{
INTRODUCTION TO THE SPECIAL ISSUE ON INTELLIGENCE ON SCALABLE COMPUTING FOR RECENT APPLICATIONS
}

\author{
P. VIJAYA*AND D BINU ${ }^{\dagger}$
}

The special issue has been focussed to overcome the challenges of scalability, which includes size scalability, geographical scalability, administrative scalability, network and synchronous communication limitation, etc. The challenges also emerge with the development of recent applications. Hence this proposal has been planned to handle the scalability issues in recent applications. This special issue invites researchers, engineers, educators, managers, programmers, and users of computers who have particular interests in parallel processing and/or distributed computing and artificial intelligence to submit original research papers and timely review articles on the theory, design, evaluation, and use of artificial intelligence and parallel and/or distributed computing systems for emerging applications. The ten papers in this special issue cover a range of aspects of theoretical and practical research development on scalable computing. The proposal provides an effective forum for communication among researchers and practitioners from various scientific areas working in a wide variety of problem areas, sharing a fundamental common interest in improving the ability of parallel and distributed computer systems, intelligent techniques, and deep learning mechanisms and advanced soft computing techniques. The issue covers wide range of applications, but with scalable problems that to be solved by perfect hybridization of distributed computing and artificial intelligence.

The first paper is "CPU-Memory Aware VM Consolidation for Cloud Data Centers" introduced a CPUMemory aware VM placement algorithm is proposed for selecting suitable destination host for migration. The Virtual Machines are selected using Fuzzy Soft Set (FSS) method VM selection algorithm. The proposed placement algorithm considers CPU, Memory, and combination of CPU-Memory utilization of VMs on the source host.

In "Bird Swarm Optimization-based stacked autoencoder deep learning for umpire detection and classification", presented the umpire detection and classification by proposing an optimization algorithm. The overall procedure of the proposed approach involves three steps, like segmentation, feature extraction, and classification. Here, the classification is done using the proposed Bird Swarm Optimization-based stacked autoencoder deep learning classifier (BSO-Stacked Autoencoders), that categories into umpire or others.

In "Enhanced DBSCAN with Hierarchical tree for Web Rule Mining", proposed an enhanced web mining model based on two contributions. At first, the hierarchical tree is framed, which produces different categories of the searching queries (different web pages). Next, to hierarchical tree model, enhanced Density-Based Spatial Clustering of Applications with Noise (DBSCAN) technique model is developed by modifying the traditional DBSCAN. This technique results in proper session identification from raw data. Moreover, this technique offers the optimal level of clusters necessitated for hierarchical clustering. After hierarchical clustering, the rule mining is adopted. The traditional rule mining technique is generally based on the frequency; however, this paper intends to enhance the traditional rule mining based on utility factor as the second contribution. Hence the proposed model for web rule mining is termed as Enhanced DBSCAN-based Hierarchical Tree (EDBHT).

In "A comprehensive survey of the Routing Schemes for IoT applications", this review article provides a detailed review of 52 research papers presenting the suggested routing protocols based on the content-based, clustering-based, fuzzy-based, Routing Protocol for Low power (RPL) and Lossy Networks, tree-based and so on. Also, a detailed analysis and discussion are made by concerning the parameters, simulation tool, and year

\footnotetext{
*Waljat College of Applied Sciences, Rusayl, Muscat, Sultanate of Oman (pvvijaya27@gmail.com)

${ }^{\dagger}$ Resbee Info Technologies, Tamilnadu, India (altimatebinu@gmail.com)
} 
of publication, network size, evaluation metrics, and utilized protocols. In "Chicken-Moth Search OptimizationBased Deep Convolutional Neural Network For Image Steganography", proposed an effective pixel prediction based on image stegonography is developed, which employs error dependent Deep Convolutional Neural Network (DCNN) classifier for pixel identification. Here, the best pixels are identified from the medical image based on DCNN classifier using pixel features, like texture, wavelet energy, Gabor, scattering features, and so on. The DCNN is optimally trained using Chicken-Moth search optimization (CMSO). The CMSO is designed by integrating Chicken Swarm Optimization (CSO) and Moth Search Optimization (MSO) algorithm based on limited error.

In "An Efficient Dynamic Slot Scheduling Algorithm for WSN MAC: A Distributed Approach", an effective TDMA based slot scheduling algorithm needs to be designed. In this paper, we propose a TDMA based algorithm named DYSS that meets both the timeliness and energy efficiency in handling the collision. This algorithm finds an effective way of preparing the initial schedule by using the average two-hop neighbors count. Finally, the remaining un-allotted nodes are dynamically assigned to slots using a novel approach.

In "Artefacts removal from ECG Signal: Dragonfly optimization-based learning algorithm for neural network-enhanced adaptive filtering", proposed a method utilizes the adaptive filter termed as the (Dragonfly optimization + Levenberg Marqueret learning algorithm) DLM-based Nonlinear Autoregressive with eXogenous input (NARX) neural network for the removal of the artefacts from the ECG signals. Once the artefact signal is identified using the adaptive filter, the identified signal is subtracted from the primary signal that is composed of the ECG signal and the artefacts through an adaptive subtraction procedure.

In "A Comprehensive Review on State-of-the-Art Image Inpainting Techniques", this survey makes a critical analysis of diverse techniques regarding various image inpainting schemes. This paper goes under (i) Analyzing various image inpainting techniques that are contributed in different papers. (ii) Makes the comprehensive study regarding the performance measures and the corresponding maximum achievements in each contribution. (iii) Analytical review concerning the chronological review and various tools exploited in each of the reviewed works.

In "An Efficient Way of Finding Polarity of Roman Urdu Reviews by Using Boolean Rules", proposed a novel approach by using Boolean rules for the identification of the related and non-related comments. Related reviews are those which show the behavior of a customer about a particular product. Lexicons are built for the identification of noise, positive and negative reviews.

The final paper is "Forecasting the Impact of Social Media Advertising among College Students using Higher Order Statistical Functions", this research work plans to develop a statistical review that concerns on social media advertising among college students from diverse universities. The review analysis on social media advertising is given under six sections such as: (i) Personal Profile; (ii) Usage; (iii) Assessment; (iv) Higher Order statistics like Community, Connectedness, Openness, Dependence, and Participation; (v) Trustworthiness such as Trust, Perceived value and Perceived risk; and (vi) Towards advertisement which involves attitude towards advertisement, response towards advertisement and purchase intension. 